\title{
Methods of Information Extraction from OCT Images
}

\author{
A. ZAJAC ${ }^{a, *}$, Ł. URBAŃSKI ${ }^{a}$, J. KASPRZAK ${ }^{b}$ AND D. KeCIK ${ }^{b}$ \\ ${ }^{a}$ Institute of Optoelectronics, Military University of Technology, S. Kaliskiego 2, 00-908 Warszawa, Poland \\ ${ }^{b}$ Department and Ophthalmology Clinic, I Medicine Faculty, Medical University of Warsaw \\ W. Lindleya 4, 02-005 Warsaw, Poland
}

\begin{abstract}
Optical diagnostic methods of biological media are more and more willingly used in clinical practice — also instead of classical biopsy. Particularly, optical coherent tomography methods and mammography (permitting achieving three-dimensional imaging of a tissue) are attractive in comparison with classical diagnostic methods. The article presents the analysis methods of optical parameters of biological tissues developed allowing obtaining absolute values of these parameters. Thanks to modern image analysis methods - wavelet transforms - the optical diagnostic effectiveness was improved. The theoretical and practical results achieved were compared with results of commercially accessible methods implemented in OCT devices.
\end{abstract}

PACS: 87.57.-s, 42.79.Qx, 42.30.Wb

\section{Introduction}

Tomography methods in medical diagnostics allow for both tissue lesions spatial localization and its size estimation. As proved by Radon, there exists a possibility to reproduce the information about the structure of a studied medium with given resolution due to a finite number of its $z$-scans [1]. A z-scan is a medium cross-section by a perpendicular plane. The set of the studied medium $z$ -scans is called a tomogram. An important classification criterion of the tomography methods in medical diagnostics is the kind of radiation used in a specific system. The very first application of a laser light in tomography was demonstrated in $1991[2,3]$. The tomograms obtained in this specific case were featured with a $30 \mu \mathrm{m}$ resolution. The method discussed was called optical coherence tomography (OCT). This specific method allows for tissues imaging with an extreme resolution. The OCT devices provided with Superluminescence LED (SLED) sources radiation (spectral range $810-830 \mu \mathrm{m}, 40 \mathrm{~nm}$ FWHM bandwidth) make it possible to examine tissues with single nanometer accuracy. Nevertheless, the OCT tomograms interpretation is often troublesome. The information yielded from OCT images can be enhanced due to certain specific procedures introduced in presented contribution. The procedures proposed allow defining the optical parameters of tissue examined (absorption, scattering and scattering anisotropy coefficients) and tissue border recognition as well. The first technique uses the comparison of the real tissue tomogram and the Monte Carlo $[4,5]$ method based model of laser-tissue interaction. The procedure of tissue border recognition is found

\footnotetext{
* corresponding author; e-mail: azajac@wat.edu.pl
}

on the wavelet analysis. The invented procedures utility was verified by means of their application to specific OCT tomograms. Both the tissue border recognition procedure and the Monte Carlo based technique are relatively fast. (The invented methods were equally tested by means of personal computer.)

\section{Monte Carlo simulation}

Evaluation of a radiation energy distribution in any arbitrary medium is relatively difficult. There exist two basic methods which make it possible to solve the laser beam propagation problem in an opaque media, that is human tissues. The first one is the four-flux method, which assumes medium regular structure (spherical symmetry of scattering centers). This assumption cannot be fulfilled in case of non-uniform media with irregularities (such as human tissues) [6]. The other method used for the solution of the laser beam propagation problem in opaque medium is the Monte Carlo method [7, 8]. Due to its probabilistic character the Monte Carlo method is more suitable for modeling of a media with random structure. The method accuracy is relatively high even for typical PC ALU applied for simulation.

The Monte Carlo method based model allows for qualitative estimation of radiation energy distribution in the absorbing and scattering medium. According to the spectral characteristics of optical media parameters, there exists a possibility to change the wavelength of virtual photon flux. Optical parameters spectral dependence of specific medium (hemoglobin at different oxygen saturation level) is shown in Fig. 2. Artificial medium optical parameters shifting in the simulation input are equivalent to incident laser beam wavelength tuning. 
Monte Carlo methods point that any physical phenomenon result can be estimated by the parameters of a certain random variable. The random variable is a result of a certain statistic experiment. Method accuracy is simply proportional to the number of experiment replications - the law of great numbers. The model presented is based on the virtual particle random walking problem on a given three-dimensional grid. The randomly walking particle is an analogue of a single photon while the three-dimensional grid is an analogue of illuminated tissue. The artificial model of a tissue is characterized by a set of specific optical parameters, namely: absorption coefficient, scattering coefficient and the scattering anisotropy coefficient (Fig. 1).

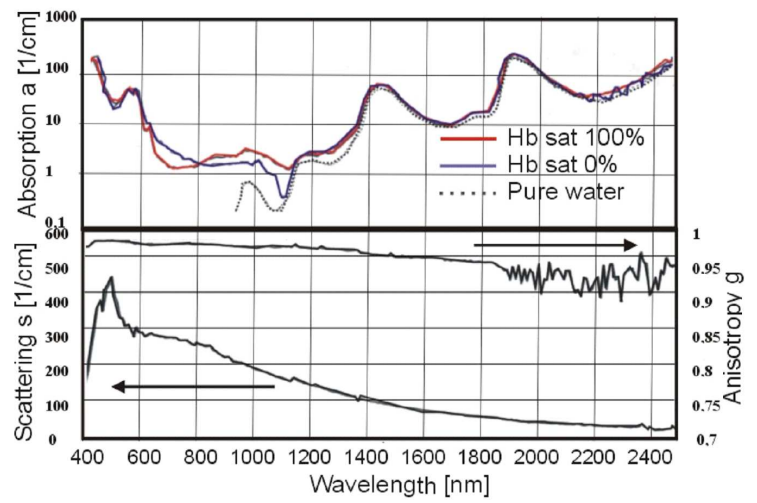

Fig. 1. The spectral characteristics of absorption coefficient (top figure), scattering coefficient and scattering anisotropy coefficient (bottom figure).

Likewise any other Monte Carlo method its accuracy enhancement is not equivocal with replication number increase. For this specific case the number of experiment repetitions was one million and was sufficient for qualitative radiation energy distribution estimation. The results of the simulation are illustrated in Fig. 2 and Fig. 3. Each picture is a cross-section of the three-dimensional medium modeled, featured by different optical parameters.

Figure 4 presents the plots of normalized laser radiation intensity versus its penetration length in a tissue. One can notice that the OCT signal is characterized by a cut-off intensity, while the simulation result is not. The cut-off intensity is conditioned by the responsitivity of the detection system applied in the specific OCT tomograph setup. The simulation cut-off intensity for the specific case presented in Fig. 4 equals 0.01 and can be set to any value from 0 to 1 by the user. The simulation and experiment results are suited to each other in the range above the cut-off intensity.

That is why there are justified premises to claim that the optical parameters of the real and the artificial medium are similar.

The optical tissue parameters estimation is achieved by comparison of the simulation and the measurement results. The measurement was taken by means of the Carl

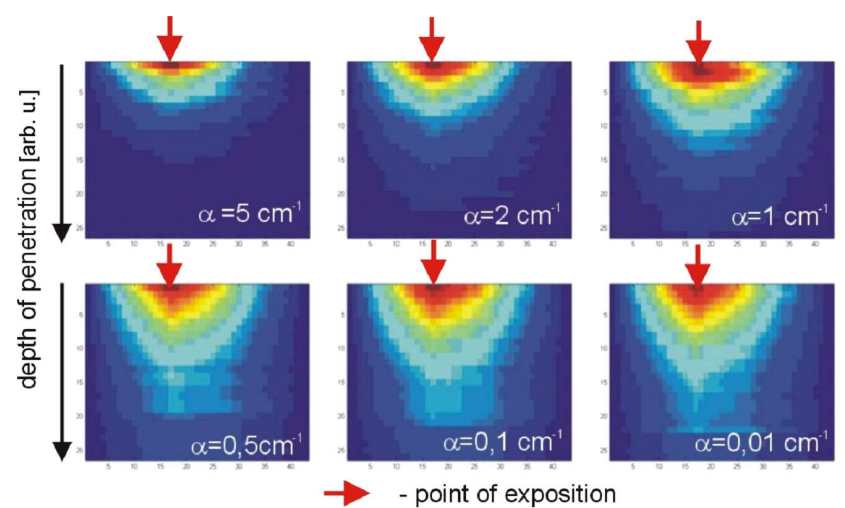

Fig. 2. Simulated laser beam energy distribution in the medium of different optical parameters (absorption coefficient $\alpha$-variable, scattering coefficient $s=15 \mathrm{~cm}^{-1}$, scattering anisotropy coefficient $g=0.9$ ).

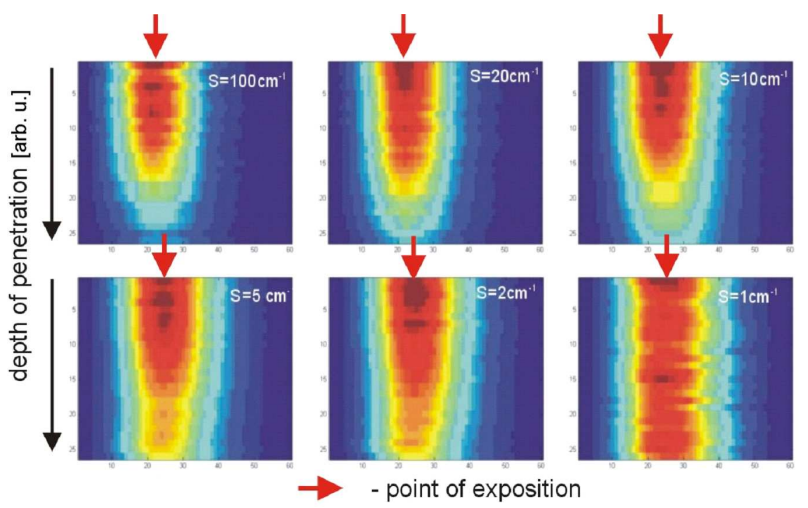

Fig. 3. Simulated laser beam energy distribution in the medium of different optical parameters (absorption coefficient $\alpha=1 \mathrm{~cm}^{-1}$, scattering coefficient $s$ - variable, scattering anisotropy coefficient $g=0.8$.

Zeiss "Stratus OCT" tomograph. The measurement data series represent one-dimensional laser radiation distribution in absorbing and scattering medium. The Monte Carlo simulation is launched for a medium characterized by specific, fixed values of optical parameters i.e. absorption coefficient, scattering coefficient, scattering anisotropy coefficient. Then the simulation result is compared with an original signal obtained via OCT tissue scanning.

\section{The wavelets based information extraction enhancement}

The OCT tomograms are a very specific group of images [9]. The retinal structure identification is often troublesome due to high resolution of OCT images. The aim of the contribution presented is to develop procedures of OCT images information extraction enhancement. A typical OCT scan is characterised by large amount of extremes in a relatively small intervals of its support. The signal of that specific property is called 


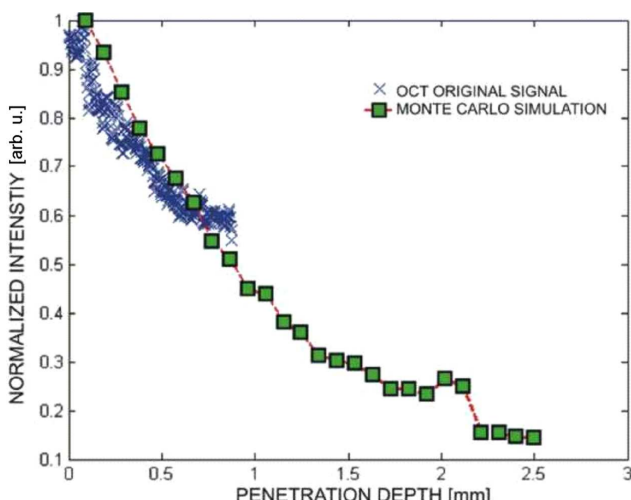

Fig. 4. Comparison of an original OCT signal with Monte Carlo simulation results.

bandwidth unlimited. The signals of these specific images can be efficiently decomposed and synthesized by means of the wavelet analysis. The procedures mentioned concern: retinal structure identification improvement, image denoising and structure boarder recognition problem. The first one employs wavelet smoothing procedure. Since wavelets generate filters characterised by finite impulse response, they can be applied to signal smoothing (Fig. 5). The operation mentioned utilizes the Haar wavelet of the fourth order.

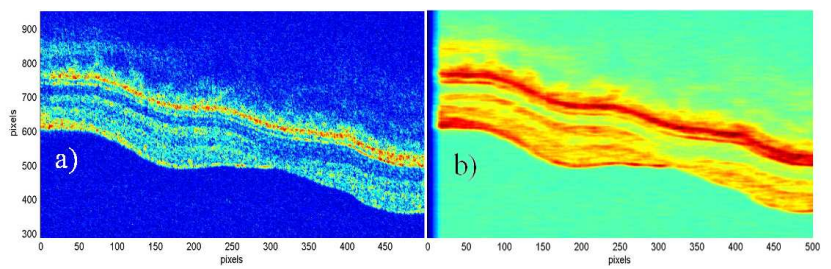

Fig. 5. The results of OCT image smoothing: OCT image of human retina before smoothing operation (a), the same image after smoothing operation (b).

Another way the wavelet analysis can be applied to OCT signal processing is the image denoising. The signal is decomposed by means of an arbitrary wavelet basis. The wavelet coefficients that are relatively small (that is do not contribute much to the signal information) are eliminated. The signal is reconstructed back with the nonzero coefficients. The relatively small wavelet coefficients characterize high spatial frequencies - susceptible to noise contribution. Figure 6 demonstrates the OCT signal decomposition of the fourth level by means of the Haar wavelet of the fourth order.

Each part of the picture presented in Fig. 6 corresponds to different image details. Counterclockwise: horizontal, diagonal and vertical. The same pattern is repeated for every decomposition level.

An additional wavelet application to OCT images processing is the boarder recognition problem. The realization of the problem is described by the graph illustrated

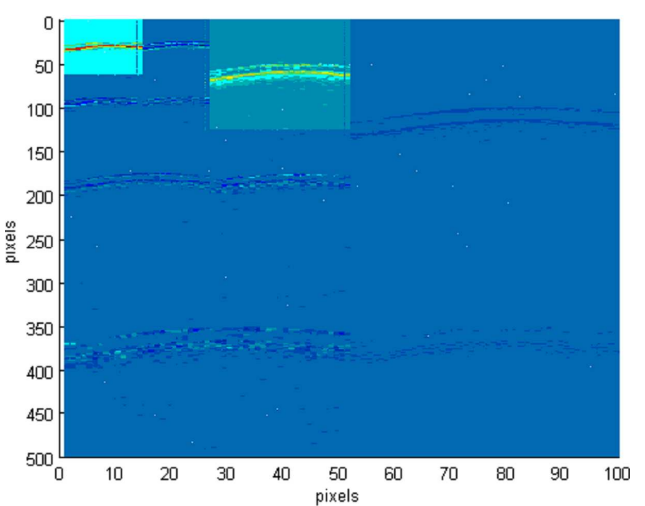

Fig. 6. Levels of OCT signal decomposition.

in Fig. 7. The very first stage of the algorithm is the OCT image smoothing by means of wavelet analysis. The second step is threshold elimination of image components of less significance. The next stage is the iterative solution of difference problem for the processed OCT image. This treatment allows for the maximum image gradient recognition. The last step of the algorithm is the polynomial approximation of the signal. The results of the described treatment are depicted in Fig. 8.

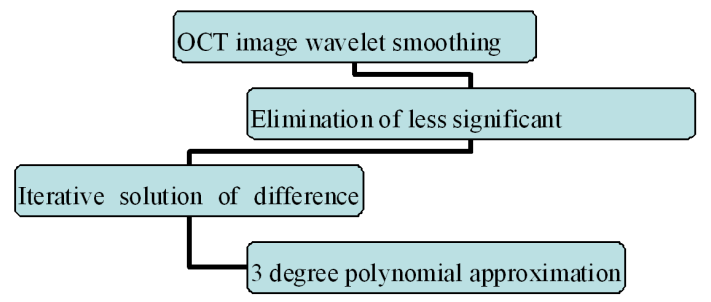

Fig. 7. The boarder recognition problem.
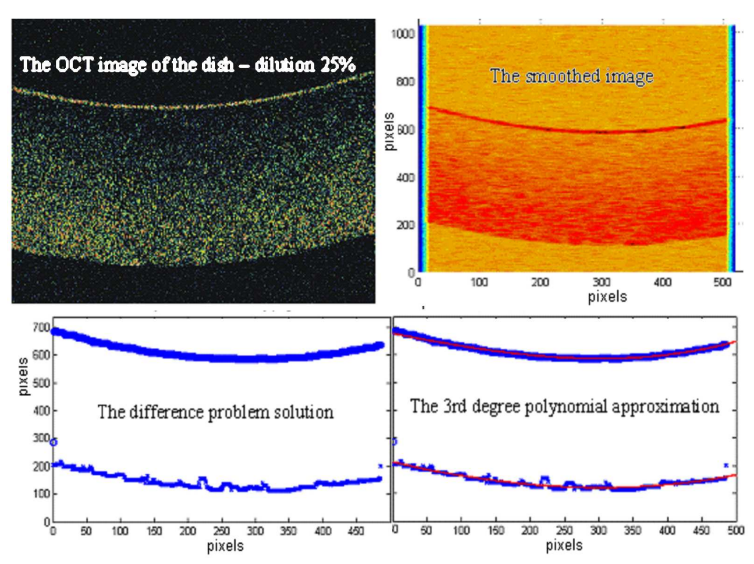

Fig. 8. The levels of the boarder recognition algorithm.

The algorithm described above was applied to different OCT images. To verify the efficiency of the algorithm media of different concentration of the scattering 
centers were measured. The tomograms taken via Carl Zeiss "Stratus OCT" system illustrate the solution of milk in water. The scattering centers concentration is changed by solution diluting with water.
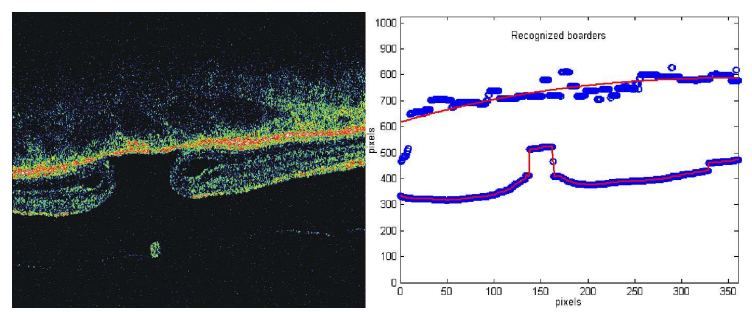

Fig. 9. The results of the boarder recognition algorithm of the pathologically changed retina. a) OCT imaging, b) results provided by the originally implemented software.

The procedure presented can be applied to retinal scans boarders recognition. The problem is of key importance concerning the retina lesions analysis. The software originally implemented by the producer cannot provide applicable results of pathologically changed retinal scans. The comparison of the results of the method invented with the results provided by the originally implemented software is shown in Fig. 9. In the case presented, instead of the 3rd degree polynomial interpolation, spine function was applied.

\section{Conclusions}

New possibilities of the OCT method application to data processing obtained during selected tissue diagnose were presented. The application of the procedures developed showed that the elimination of errors occurring in typical (commonly used) image processing is possible (Fig. 10). The analysis of a spatial radiation distribution in a tissue region exposed to a laser light beam also permits obtaining the information about absolute values of tissue optical parameters.

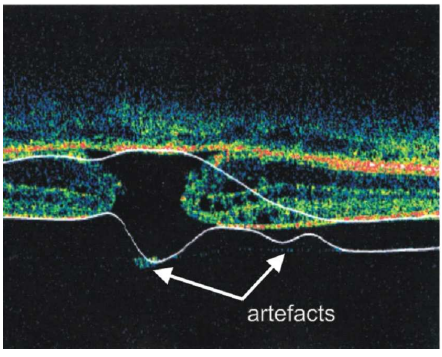

Fig. 10. The example result of incorrect recognized edges obtained by means of software of "Stratus OCT" - Carl Zeiss "Stratus OCT" tomograph device.

\section{References}

[1] R. Deans, R. Stanley, The Radon Transform and Some of Its Applications, Wiley, New York 1983.

[2] S.A. Boppart, W. Luo, D.L. Marks, K.W. Singletary, Breast Cancer Res. Treat. 84, 85 (2004).

[3] J.C. Hebden, H. Rinneberg, Physics in Medicine and Biology, Wiley, Amsterdam 2005.

[4] S.A. Prahl, M. Keijzer, S.L. Jacques, SPIE Institutes Series IS 5, 102 (1989).

[5] M. Gawlikowski, T. Pustelny, B. Przywara-Chowaniec, J. Nowak-Gawlikowska, Acta Phys. Pol. A 118, 1124 (2010).

[6] E. Hillman, Ph.D. Thesis, Department of Medical Physics and Bioengineering, London University 2002.

[7] L.G. Henyey, J.L. Greenstein, Astrophys. J. 93, 70 (1941).

[8] R. Huber, M. Wojtkowski, J.G. Fujimoto, Opt. Expr. 13, 10523 (2005).

[9] J.M. Herrmann, M.E. Brezinski, B.E. Bouma, S.A. Boppart, C. Pitris, J.F. Southern, J.G. Fujimoto, Fertil. Steril. 90, 255 (2008). 